\title{
Evolutionary Algorithms and the Vertex Cover Problem
}

\author{
P. S. Oliveto, J. He and X. Yao
}

\begin{abstract}
Experimental results have suggested that evolutionary algorithms may produce higher quality solutions for instances of Vertex Cover than a very well known approximation algorithm for this NP-Complete problem. A theoretical analysis of the expected runtime of the $(1+1)$-EA on a well studied instance class confirms such a conjecture for the considered class. Furthermore, a class for which the $(1+1)$-EA takes exponential optimization time is examined. Nevertheless, given polynomial time, the evolutionary algorithm still produces a better solution than the approximation algorithm. Recently, the existence of an instance class has been proved for which the $(1+1)$-EA produces poor approximate solutions, given polynomial time. Here it is pointed out that, by using multiple runs, the $(1+1)$-EA finds the optimal cover of each instance of the considered graph class in polynomial time.
\end{abstract}

\section{INTRODUCTION}

Evolutionary Algorithms (EAs) are randomized search heuristics that have been widely used for solving combinatorial optimization problems since the seventies [1]. However, the field analysing the time complexity of EAs is fairly new, spawning in the nineties with the analysis of the basic $(1+1)$ EA on simple pseudo-boolean functions [2]. These functions were artificially created to understand what characteristics of a problem may make its optimization easy or hard for an EA. The behaviour of the $(1+1)$-EA on toy problems such as Onemax [2], Trap Functions [3] or on plateaus of constant fitness [4] was examined. Apart from proving if the algorithm is efficient or not in optimising the problems, these efforts have led to the introduction of a different range of techniques [5] and to the design of a general Markov chain framework [6] for the analysis of EAs. Building up on this first basis, nowadays it is possible to analyse the (1+1)EA for combinatorial optimization problems with "practical" applications, such as maximum matching [7], the minimum spanning tree problem [8], the partition problem [9] and the subset sum problem [10]. In these works a new technique, Drift analysis [10], [11] has proved to be a useful tool, often (but not only) for finding exponential lower bounds.

In this paper the $(1+1)$-EA is analysed for Vertex Cover, a well known NP-Complete combinatorial optimization problem having practical applications in fields such as networking and scheduling. Experimental studies have shown that EAs are successful on Vertex Cover instances [12]. In particular, the empirical results suggest that they can find better approximate solutions than those found by a very well known approximation algorithm for Vertex Cover problems (i.e. VERCOV [13]), although with higher expected optimization

P. S. Oliveto, J. He and X. Yao are with the Centre of Excellence for Research in Computational Intelligence and Applications (CERCIA), School of Computer Science, University Of Birmingham, Edgbaston, Birmingham, B15 2TT U.K. e-mail: \{P.S.Oliveto, J.He, X.Yao\}@cs.bham.ac.uk. times. It is not sure if the above conjecture is true and nevertheless how good the solutions are, and especially what time the EA takes to find such solutions which are supposedly better.

On one hand, Bäck and Khuri [12] show experimentally that a Genetic Algorithm (GA) performs very well on instances of sizes $n=100$ and $n=202$ of the PapadimitriouSteiglitz (PS) graph [13] since it finds the optimal cover on average 6 times out of 10 in a runtime of $\mathrm{cn}^{2}$.In the remaining runs it only reaches a local optimum. VERCOV never finds the optimal cover of this graph. This problem seems to be simple for the GA, although it is not clear what polynomial time is to be expected as the graph size grows.

On the other hand, in [14] an instance class (i.e similar to a "path" [7]) is presented, for which a GA never finds the optimum in all of the 10 runs of 10000 generations each, with the graph size being $n=101$. At a glance this problem seems to be hard for the GA, and the interest is turned to whether the EA takes polynomial or exponential expected time to find the minimum cover.

This paper contains an analysis of the $(1+1)$-EA on the PS instance class and on a wider class containing the "path" instance. It is shown that the (1+1)-EA optimises the PS graph in polynomial time if multiple runs are used, although the average expected time is exponential in the graph size $n$. Concerning the other instance, instead, the runtime is exponential in $n$, even if multiple runs are used. Nevertheless, through a theoretical comparison we conclude that, given the (1+1)-EA polynomial time, it provides higher quality solutions than VERCOV on the examined instance classes of the vertex cover problem even by using single runs.

Recently a bipartite instance class has been analysed, giving an exponential expected runtime for the $(1+1)$-EA to produce an approximation which is better than the one produced by VERCOV [15]. Here it is discussed that, with a probability higher than $\frac{1}{2 e}$, the $(1+1)$-EA finds the optimal cover in $O(n \log n)$. Hence again, although by using multiple runs, the $(1+1)$-EA finds a better approximation than VERCOV on the instance class.

The paper is structured as follows. Section II introduces the Vertex Cover problem and the algorithms considered in this research paper. The Papadimitriou-Steiglitz graph is analysed in section III. The "exponential time" instance class is examined in section IV. The bipartite instance class is discussed in section V. In section VI we draw our conclusions.

\section{PRELIMINARIES}

Vertex Cover is a well known combinatorial optimization problem. Given an undirected graph $G=(V, E)$, with $V$ being the set of vertexes, or nodes, and $E$ the set of edges, 
the Vertex Cover problem is that of finding the minimum subset $C$ of $V$ such that for any edge $e \in E$ at least one of its endpoints is in $C$. All the subsets $C$ of $V$ having, for each edge $e \in E$, at least one of its endpoints in $C$ are called covers. All the other subsets of $V$ are infeasible. Since Vertex Cover has been proved to be NP-Complete [13], the current goal of researchers is to find approximation algorithms that in polynomial running time return near-optimal solutions. A well known algorithm for Vertex Cover is VERCOV, a 2-Approximation algorithm for the problem [13]. This means that whatever the graph instance, VERCOV returns a solution which is at most twice the size of the optimal cover. VERCOV, starts with an empty cover and randomly chooses an edge $(u, v)$. Then it deletes all the edges incident to either $u$ or $v$ and inserts the two endpoints in the cover. The process is repeated until all the edges are removed from the graph.

In this paper VERCOV will be compared with a Random Local Search (RLS) algorithm which flips one bit per iteration, and with a basic evolutionary algorithm, the $(1+1)$ $\mathrm{EA}$, on some empirically well-studied instance classes of the Vertex Cover problem. The $(1+1)$-EA flips each bit with probability $1 / n$ and uses elitist selection. Different initialisations are examined, varying from an initial empty cover, a uniform distribution and an initial full cover, with the hope of getting an idea of whether one should be preferred to the others.

For the (1+1)-EA to be adapted to optimise a vertex cover instance, each subset $C \in V$ is represented by a bit-string $\left(s_{1} \ldots s_{n}\right)$ with $n$ being the number of vertices of the graph $G=(V, E)$. For each node $v_{i}$ belonging to the subset $C$ the relative bit $s_{i}$ is set to 1 . Otherwise it is set to 0 . Given the above representation a fitness function for the Vertex Cover can be introduced as in [12] and [14]:

$$
f(C)=\sum_{i=1}^{n}\left(s_{i}+n\left(1-s_{i}\right) \sum_{j=1}^{n}\left(1-s_{j}\right) e_{i, j}\right)
$$

Here the first part of the formula counts the number of nodes in the cover and the second part gives a penalty to each uncovered edge $e$. As a result, any cover has a better fitness value than that of an infeasible solution.

\section{AN INSTANCE CLASS WITH POLYNOMIAL TIME}

In this section the performance of the $(1+1)$-EA is analysed on the Papadimitriou-Steiglitz graph [13] which was previously studied empirically in [12]. The graph, and its optimal cover with $k=3$, is shown in figure 1 . The experimental results gave hints that genetic algorithms perform better than VERCOV on this instance class. Here we prove that the average expected time for the (1+1)-EA to reach the optimum is exponential, but multiple runs of the algorithm lead to an expected optimisation time of $O(n \log n)$. The same holds for RLS. On the other hand VERCOV always finds a suboptimal cover which is worse than the one found by the $(1+1)$-EA in time $O(n \log n)$, even if the EA is run only once.

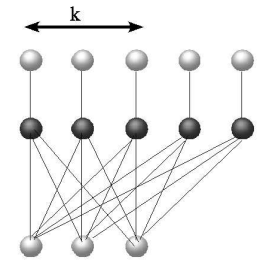

Fig. 1. The optimal cover for the Papadimitriou-Steiglitz graph with $k=3$. The total number of nodes is $n=3 k+4$.

Theorem 1: The expected time for RLS to optimise the Papadimitriou-Steiglitz graph, starting with a full cover, is infinite. With probability at least $\frac{1}{4}$ RLS finds the global optimum in expected time $O(n \log n)$.

Proof: With probability $\frac{k}{2 k+2}>\frac{1}{4}$ a node of the third row of the graph is removed from the cover before any node of the second row. If this event happens, then no bit flips of second row nodes will be accepted. When all the nodes of the first and the third row have been selected at least once they will have all been removed and the global optimum will have been found. The expected time for this to happen is $O(n \log n)$, which is obtained with a similar analysis as that of RLS on Onemax [16]. On the other hand, with a probability of $\frac{k+2}{3 k+4}$ a second row node is the first node to be removed from the cover. In such a case the worst local optimum (i.e. the opposite of the optimal cover) will be found and the algorithm will be trapped forever since it does not flip more than one bit at a time.

Theorem 2: The expected time for the $(1+1)$-EA to optimise the Papadimitriou-Steiglitz graph is $2^{\Omega(n)}$. With probability at least $\frac{1}{7 e}$ the $(1+1)$-EA finds the global optimum in expected time $O(n \log n)$.

The theorem points out that, although the expected time is exponential given one run, by using multiple runs the $(1+1)$ EA surely finds the optimal solution in polynomial time. Lemmas 1 and 3 show how, according to the initialisation, the algorithm finds the solution quickly if multiple runs are used (i.e. in expected time $O(n \log n)$ ). Lemma 5 proves that in the worst case the expected time is exponential in $n$.

Lemma 1: If the $(1+1)-\mathrm{EA}$ is initialised with all the nodes in the cover, with probability at least $\frac{1}{7 e}$, the $(1+1)$-EA finds the global optimum in expected time $O(n \log n)$.

Proof: If the EA is initialised with all the nodes in the cover, it only accepts bit flips reducing the cover size or creating a different cover of the same size as the current one.

The proof consists of two parts. The first part proves that with a probability higher than $\frac{1}{7}$ at least a node of the third row of the graph is removed from the cover before any of the middle row nodes. The second part shows that if such an event happens, then the (1+1)-EA will find the global optimum with a probability of at least $\frac{1}{e}$.

For the first part, the probability that no bits flip can be ignored since it is not influential. The conditional probability that exactly one bit flips given that at least one bit flips is $\frac{P(1-\text { bitflip })}{1-P(0-\text { bitflip })} \geq \frac{1 / e}{1-1 / 2 e}=\frac{2}{2 e-1}$. 
Given that a 1-bit flip occurs, the probability that a third row node is chosen rather than a second row node is at least $\frac{1}{3}$. Hence, $\frac{2}{2 e-1} \frac{1}{3} \geq \frac{1}{7}$ is a lower bound on the probability that at least one node of the third row is selected before a second row node. If a bit belonging to the third row of the graph is chosen in the first step, then any 1-bit flip concerning a node in the central row (i.e. all the nodes of the global optimum) will not be accepted since one of the edges between the second and the third row would become uncovered, hence the new solution would not be a cover.

Concerning 2-bit flips, if the removed bit is chosen together with a central row bit, then the new individual will be accepted. Let such an event be $E_{2}$. Being the central row nodes, $k+2$, the expected time for $E_{2}$ to happen is at least $\Omega(n)$ since $P_{2}=(k+2) \frac{1}{n^{2}}\left(1-\frac{1}{n}\right)^{n-2} \leq \frac{1}{e(n-1)}$. Since in expected time $O(1)$ ( being $P_{1} \geq \frac{k-1}{e n}$ ), another third row bit is removed from the cover (event $E_{1}$ ), the above 2-bit flip case does not happen in a typical run of the algorithm. Once two nodes belonging to the third row have been chosen, hence removed, at least three bits have to be chosen for a central row node to be removed from the cover. These three bits should refer to the two already removed nodes of the third row and to any one of the central row nodes (event $E_{3}$ ). The expected time for one of the described combinations to be chosen is $\Omega\left(n^{2}\right)$. The rest of the proof relies on the fact that with probability at least $\frac{1}{e}$ such events do not happen before the algorithm finds the global optimum. The probability that $E_{1}$ occurs before $E_{2}$ is at least

$$
\frac{P_{1}}{P_{1}+P_{2}} \geq \frac{k-1}{e n} /\left(\frac{k-1}{e n}+\frac{1}{e(n-1)}\right) \geq 1-\frac{2}{k} .
$$

Since the probability of event $E_{2}$ is higher than that of any other event $E_{i}$ with $2<i \leq k-1$, this is a lower bound on the probability of $E_{1}$ happening before any event $E_{i}$. The $(1+1)$-EA has to remove other $k-1$ third row nodes from the cover before any event $E_{i}$ may happen. $\left(1-\frac{2}{k}\right)^{\frac{k}{2}-1} \geq \frac{1}{e}$ is a lower bound for the probability that any event $E_{i}$ may happen before $\frac{k}{2}$ third row nodes have been removed from the cover. Once they have been removed, the probability of a middle row node being removed from the cover is $O\left(n^{-\left(\frac{k}{2}-1\right)}\right)$ which is exponentially small. In the mean time the first row nodes that are still in the cover, if any, will be removed. Similarly to the analysis of ONEMAX the expected time for this to happen is $O(n \log n)$. The proof of the lemma follows.

If, instead, the first node to be removed from the cover belongs to the first row, then the conditional probability of reaching the global optimum is higher. This occurs because the removal of the row 1 node, blocks the node on the same position in row 2. On the other hand, if the first node to be removed from the cover belongs to the second row, then it is the nodes in the third row that are passive to 1-bit flips, hence blocked. In the considered case, with the nodes in the third row being blocked, only those belonging to the other two rows may be removed from the cover by flipping 1-bits. If a node in position $i$ of either of the two rows is removed from the cover, then the node in the same column but in the other row, will be blocked. Hence, the worst solution that is obtained by just using 1-bit flips contains all the $k$ nodes of the third row and $k+2$ nodes randomly chosen from both the first and the second row. Such a cover is called a local search cover. Figure 2 shows different local search covers for a graph with $k=3$. With similar calculations as in lemma 1 the above discussion proves the following lemma:

Lemma 2: RLS generates a cover of at most $2 k+2$ nodes in expected time $O(n \log n)$.

Lemma 3: If the $(1+1)-E A$ is initialised with an empty cover, then with a probability higher than $\frac{1}{3 e}$, the global optimum is found in expected time $O(n \log n)$.

Proof: The proof relies on a probability of at least $\frac{1}{3}$ that, given an initial empty cover, all the nodes of the central row are inserted in the cover before all the nodes of the third row are. The nodes of the first row do not influence the process since each node of the last row is connected with all the middle row nodes. This implies that in every possible cover, either at least all the third row nodes belong to a cover, or at least all the middle row ones do. Otherwise the solution is not a cover.

If all the second row nodes are inserted in the cover before all of the third row ones, then from lemma 1 with a probability of at least $\frac{1}{e}$ the optimum is found in expected time $O(n \log n)$ and the proof follows.

Corollary 1: With probability at least $\frac{1}{3}$ RLS finds the global optimum in expected time $O(n \log n)$, if the algorithm is initialised with an empty cover.

If the $(1+1)$-EA does not find the global optimum it will reach a local search cover in time $O(n \log n)$. The worst local search cover (fig.2(a)) is that having all the $k+2$ first row nodes in the cover, hence none of the second row. In this case the distance from the global optimum is maximised since, in order to obtain the optimal solution, all the nodes in the cover have to be removed and all the others need to be inserted. The best local search cover is that having all the second row nodes in the cover but one (fig.2 (d)). In this case the distance to the global optimum is minimised.

Lemma 4: If the (1+1)-EA finds a local search cover, then it will take time $O(n \log n)$ and with overwhelming probability it will have at most $\frac{2}{3}(k+2)$ middle row nodes.

Proof: Since we are assuming a local search cover will be found, then all the third row nodes will be in the cover. The process terminates when they are in the cover together with either a first row node or a middle row one for each of the $k+2$ columns. The proof that the expected time is $O(n \log n)$ is similar to that of lemma 1 . Let the process start with a full cover. For each column, the conditional probability that a first row node is removed from the cover rather than a middle row one, given that a node of the column gets removed, is $P\left(\right.$ first_row column $\left._{i}\right)=\frac{1}{2}$. We consider $N=k+2$ trials, one for each column, and estimate the number of times a first row node is chosen. By using Chernoff bounds it follows that the probability that more than $\frac{2}{3} N$ first row nodes are chosen is overwhelmingly low. 
a)

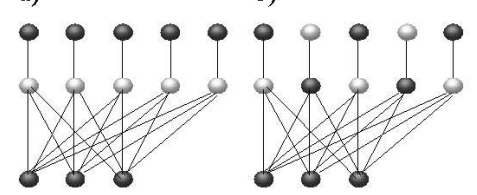

c)

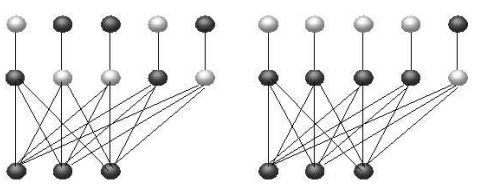

Fig. 2. a) The worst local search cover for the Papadimitriou-Steiglitz graph with $k=3$. b) and c) are different local search covers: all the local search covers, not being the optimal one, have all the third row nodes in the cover, and one node from either of the other two rows for each column. d) The best local search cover with $k=3$.

The proof when the algorithm is initialised with an empty cover is similar.

Lemma 5: The expected time for the $(1+1)$-EA to reach the global optimum from a local search cover with at most $\frac{2}{3}(k+2)$ middle row nodes is at least $2^{\Omega(n)}$.

In the following the proof idea is given. A complete drift analysis proof can be found in [17].

Proof: If the current solution is a local search cover with at most $\frac{2}{3}(k+2)$ middle row nodes, then for at least a node belonging to the third row to be removed from the cover it is necessary that at least all the central row nodes that are not in the cover get flipped together with the same number of third row nodes. The expected time for such an event to happen is at least $\Theta\left(n^{\frac{4}{3}(k+3)}\right)$ which is exponential in $n$. Hence the case of the first row nodes that are exchanged gradually with those of the middle row should be examined for the algorithm to reach the global optimum. When all the nodes of the second row have been exchanged with those of the first one, then the third row nodes will quickly be removed from the cover by flipping one bit at a time.

For an exchange of cover nodes between the first two rows at least two bits need to flip, one for each row in the same column. Let a move that is accepted be a successful move and let $N=k+2$ be the number of nodes in each of the first two rows.In the following we will consider the time it takes to get from $\frac{2}{3} N$ middle row nodes in the cover to $\frac{3}{4} N$ middle row nodes and use ideas from the Gambler's ruin problem [18] to prove that the expected time is exponential in $n$.

Let $S_{\frac{1}{4} N}$ be the state when the current solution is a cover with $\frac{3}{4} N$ middle row nodes and $\frac{1}{4} N$ first row nodes. Hence this is the point of the search space we will prove takes exponential time to be reached. Let $S_{\frac{1}{3} N}$ be the state when the current cover is a cover with $\frac{2}{3} N$ middle row nodes and $\frac{1}{3} N$ first row nodes. We are optimistically assuming the process starts from a local search cover half way between the states $S_{\frac{1}{3} N}$ and $S_{\frac{1}{4} N}$. As proved in the previous lemma the probability that the process starts here is exponentially low, and the probability of a jump from state $S_{\frac{1}{3} N}$ to a point in the middle is exponentially small and also are the probabilities of jumping to a nearer point to the optimum than state $S_{\frac{1}{4} N}$.

Now we will calculate the probability that state $S_{\frac{1}{3} N}$ is reached before state $S_{\frac{1}{4} N}$, hence the probability the process moves in the wrong direction. The following calculations only consider 2-bit flips to give an idea of the reasons for the exponential time bound. We will consider the gambler wins when he reaches state $S_{\frac{1}{3} N}$, while he is ruined when he reaches state $S_{\frac{1}{4} N}$. Then the time to reach the end of the considered space is the expected time for the gambler to lose rather than win. The total capital is $c=\frac{1}{3} N-\frac{1}{4} N=\frac{1}{12} N$ dollars and the initial capital of the gambler is half the total capital, hence $a=\frac{1}{24} N$ dollars.

The conditional probability in state $S_{\frac{1}{3} N}$ of the gambler losing (i.e. getting a better cover) is $P\left(S_{\frac{1}{3} N}^{-} \mid\right.$successful $)=$ $\frac{(1 / 3) N}{N}=1 / 3$, since there are $\frac{1}{3} N$ 2-bit flips leading to an extra middle node in the cover out of $N$. The probability of exchanging a middle row node of the cover with a first row one, hence of the gambler winning is $P\left(S_{\frac{1}{3} N}^{+} \mid\right.$success ful $)=$ $\frac{(2 / 3) N}{N}=2 / 3$. In the rest of the space between $S_{\frac{1}{4} N}$ and $S_{\frac{1}{3} N}$ the probability $P^{-}$of moving towards the optimum is lower than $P\left(S_{\frac{1}{3} N}^{-} \mid\right.$successful $)$while the probability of moving in the opposite direction, $P^{+}$, is higher than $P\left(S_{\frac{1}{3} N}^{+} \mid\right.$successful $)$. So if we assume $p=2 / 3$ and $q=1 / 3$ we are considering a probability of the gambler winning which is lower than that of the actual process.

The probability that the gambler wins is [18]:

$$
\frac{1-t^{a}}{1-t^{c}}
$$

where $t=q / p=1 / 2$. It can be noticed that for $p>1 / 2$ the above probability is at least $1-t^{a}$. By applying the formula we get that the probability of the gambler winning is at least $1-t^{a}=1-\left(\frac{1}{2}\right)^{(1 / 24) N}$. So the probability of the gambler losing is at most $\left(\frac{1}{2}\right)^{(1 / 24) N}=2^{-\Omega(n)}$. Hence the expected number of times the process has to be repeated before the algorithm reaches the end of the phase is $2^{\Omega(n)}$.

From lemma 4, only with overwhelmingly low probability the algorithm reaches a local search cover with more than $\frac{2}{3} N$ second row nodes. From such a position lemma 5 shows that with overwhelming probability exponential time is expected for the $(1+1)$-EA to reach the global optimum. So lemmas 1, 3, 4 and 5 prove theorem 2 .

Theorem 3: VERCOV always finds a cover of size $2 k+4$ on the Papadimitriou-Steiglitz graph.

Proof: The proof is divided into three points.

1) All the nodes of the central row of the graph have to be in the cover. This is because each node in row 1 is connected only with a node of row 2 of the same column. The proof of point 1 follows by considering 


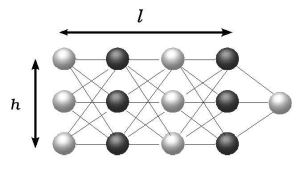

Fig. 3. The optimal cover for the $G_{3,4}$ graph.

that VERCOV can not insert a node of row 1 without inserting the node of row 2 in the cover as well.

2) For each central node in the cover another node is also inserted belonging to a different row. This follows by considering again that VERCOV inserts two adjacent nodes at a time in the cover, and that no nodes belonging to the central row are connected with each other.

3) There are no nodes $i$ and $j$, neither of them belonging to the central row and connected to each other by an edge.

Points 1. and 2. are sufficient to prove that the cover generated by VERCOV is at least of size $2 k+4$, since the nodes in row 2 are $k+2$. Point three proves that the cover is at most of size $2 k+4$ since once there are no edges left VERCOV returns the result.

Theorems 1 and 2 show that, by using multiple runs the (1+1)-EA and RLS always find the global optimum in polynomial time $O(n \log n)$, while, as proved in theorem 3, VERCOV always finds a larger cover. Furthermore, theorems 2 and 3 also show that, even in those runs when the $(1+1)$-EA and RLS take exponential optimization time, after $O(n \log n)$ steps they still find a better solution than VERCOV on the considered instance class.

\section{AN INSTANCE CLASS WITH EXPONENTIAL TIME}

This section introduces a class of graphs with expected exponential time for the $(1+1)$-EA. Each graph in the instance class, $G_{h, l}$ depends on two parameters, $l$ and $h$ both integers, with $n=l * h+1$ being the total number of nodes, and $l$ is even. Each of the $l * h$ nodes is uniquely defined by a couple (i,j) with $1 \leq i \leq h$ and $1 \leq j \leq l$. The remaining node is called the single node. Each node $(\mathrm{i}, \mathrm{j})$ is connected by an edge to the nodes $(i, j-1)$ and to the nodes $(i, j+1)$, with $1 \leq i \leq h$. The single node is connected with the nodes $(1, l)$, $(2, l), \ldots(h, l)$. The unique optimal cover of a graph $G_{h, l}$ has $\frac{n-1}{2}$ nodes, while $\frac{n+1}{2}$ is the number of nodes belonging to an almost optimal cover (i.e. the fittest local optimum which is not global). Figure 3, depicts a graph $G_{3,4}$ and its optimal cover. In the following it will be shown how the $(1+1)$-EA takes exponential expected time to optimise graphs belonging to the described instance class and how it is parameter $h$ that makes the problem hard for the algorithm rather than $l$ which, as a reminder, is even.

Theorem 4: The $(1+1)$-EA finds a cover of at most $\left\lfloor\frac{2}{3} l\right\rfloor h+1$ nodes in expected time $O\left(n^{2}\right)$.

The conjecture behind the theorem is that the algorithm quickly reaches a suboptimal cover even by using one-bit flips only. Flips of higher number of bits may only speed up

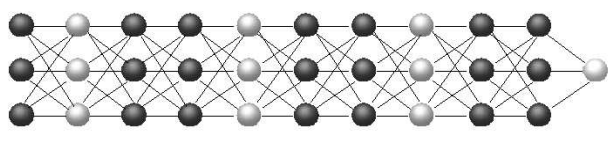

Fig. 4. The worst local search cover for the $G_{3,10}$ graph.

the process if they are accepted. The obtained cover is called the local search cover, since a local search algorithm would also find a cover of the same size.

Lemma 6: If the $(1+1)-\mathrm{EA}$ is initialised with all the nodes in the cover, then the expected time to reach a cover of size at most $\left\lceil\frac{2}{3} l\right\rceil h$ is $O(n \log n)$.

Proof: A node belonging to the cover is said to be blocked if the selection operator does not accept a 1-bit flip of such a node (i.e. by flipping the bit, the new solution would not be a cover anymore). Otherwise the node is said to be free (i.e. by flipping the bit the new solution is a cover of smaller size). Given such definitions, the following properties for covers of the instance class graph can be described:

1) Each node that is not present in the cover blocks $2 h$ nodes of the cover: the whole column on the left of the node and the whole column on its right. The single node and the nodes belonging to the far left column make an exception by blocking only $h$ nodes each.

2) A node $i$ is free only if all the nodes in the columns at its right and at its left belong to the current cover. Since all the nodes in a column are connected with the same nodes of the adjacent columns, if a node is not in the current cover then all the nodes of its column are free.

3) A column is blocked if at least one node belonging to one of its adjacent columns does not belong to the current cover.

By considering the three properties above, it is easy to see that, given a cover of the graph, in every group of three adjacent columns at most two can be blocked, hence one must be free (i.e. If the three adjacent columns are all in the cover then the nodes in the middle column are obviously free). It follows that there may be at most $\left\lceil\frac{2}{3} l\right\rceil$ blocked columns in total. It is worthy to point out that the first two columns of the graph can not both be blocked at the same time, because if the second column is in the cover then the first is free. Similar reasoning applies for the last two columns because, although the single node blocks the last column, a 2-bit flip is sufficient to make it free. This last 2-bit flip is what makes the expected time $O\left(n^{2}\right)$ rather than $O(n \log n)$ to reach a cover size of at most $\left\lfloor\frac{2}{3} l\right\rfloor h+1$.

Hence, the selection operator of the $(1+1)$-EA will surely accept 1-bit flips as long as the number of nodes in the cover is larger than $\left\lceil\frac{2}{3} l\right\rceil h$. With a proof similar to that of the $(1+1)$ EA for ONEMAX, an upper bound of $O(n \log n)$ can be obtained for the expected time to reach, at least, any local search cover. Only 1-bit flips are considered, since flips of higher number of bits may exchange the columns being free and being blocked, or may speed up the described process. 
In any case they will never augment the cover size.

Lemma 7: If the $(1+1)-\mathrm{EA}$ is initialised with an empty cover, then the expected time to reach a cover of size at most $\left\lceil\frac{2}{3} l\right\rceil h$ is $O(n \log n)$.

Proof: Let a node not belonging to the cover be blocked, if all its edges connect it with nodes belonging to the cover. Otherwise let the node be free. The algorithm, starting with an empty set, adds any free node it randomly chooses to the cover set until there are no more free nodes. In such a case a cover has been reached because all the remaining nodes that do not belong to the current cover-set are blocked.

At the beginning of the process all the nodes are free, since the cover is empty. As the process goes on, when a column has all the column at its right and all the column at its left in the cover, then it is completely blocked. The described process will have a maximum length when all the nodes but one are accepted before a cover is reached (i.e. the columns are filled from right to left or from left to right). In such a worst-case event, all the nodes but one will be inserted in the cover, hence they have to be chosen. This case can be compared to the worst case of the ONEMAX problem, when the process starts with a bit string of all zeros and has to be turned to a bit string of all ones. The only difference is that the process surely terminates when there is only one zero-bit left at most, hence the expected time is a little lower. So the upper bound of $O(n \log n)$ for the (1+1)-EA on ONEMAX [16] is valid for the expected time of the algorithm to reach a cover in this case. When, instead, some columns or nodes get blocked, the process will terminate earlier and the $O(n \log n)$ upper bound still holds.

As stated above, in the worst case the cover that is reached has at most $n-1$ nodes. From such a cover, lemma 6 guarantees an upper bound of $O(n \log n)$ on the expected time for the algorithm to reach a local search cover. The proof of the lemma follows. Again higher number of bits flipped per generation may only speed up the process.

Lemma 8: If the $(1+1)$-EA is initialised with a uniform distribution, then the expected time to reach a cover of size at most $\left\lceil\frac{2}{3} l\right\rceil h$ is $O(n \log n)$.

Proof: By initialising the EA with a uniform distribution, before the evolutionary process begins, each node is inserted in the cover set with probability $p=1 / 2$. The resulting set of nodes may or may not be a cover. If it is a cover then the proof uses similar arguments to those of lemma 6. Otherwise arguments of lemma 7 are used.

The proof of theorem 4 follows from lemmas 6,7 and 8 , and by considering that if the EA gets trapped in a figure 4 pattern it takes $O\left(n^{2}\right)$ time to remove the nodes of the last column on the right by replacing them in the cover with the single node.

Corollary 2: RLS finds a cover of at most $\left\lceil\frac{2}{3} l\right\rceil h$ nodes in expected time $O(n \log n)$.

Theorem 5: The $(1+1)$-EA has an exponential expected runtime $\Omega\left(n^{2 \sqrt{n-1}}\right)$ on $G_{h, l}$ if $h=l$ and $\Omega\left(n^{2 h}\right)$ if $l$ is a constant.

Theorem 4 proves that at least a local search cover is found in time $O(n \log n)$. The following three lemmas prove that with exponentially low probability it is the global optimum.

Lemma 9: If the algorithm is initialised with all the nodes in the cover, then with overwhelmingly high probability, the $(1+1)$-EA does not find the global optimum before it finds a local search cover.

Proof: For the global optimum to be found, all the nodes belonging to columns $i$, with odd $i$, have to be removed from the cover. First the probability that this happens, before any even column nodes are removed, will be derived. After, it will be proved that if an even column node is removed, then the probability of reaching the global optimum before another local search cover is even lower.

Starting with a full cover the probability of choosing an odd column node or the single node by flipping one bit is $P_{0}=\frac{\frac{l h}{2}+1}{l h+1}=\frac{1}{2}+\frac{1}{2 n}$. The probabilities of accepted flips of higher number of bits choosing only odd column nodes are lower. If the most "convenient" odd column is chosen then four columns are blocked (i.e. if a node of the third column is removed, then if nodes of the second and the fourth column were to be chosen, the flips would not be accepted. Hence the first column nodes are destinated to be removed also), so at least $\frac{1}{4} l h$ different column nodes have to removed before 1-bit flips will guarantee finding the global optimum. It is optimistically assumed that if an even column is blocked, then flips of higher number of bits do not change the situation. At the next step the probability of choosing another odd column node from a different column out of $X_{1} \geq l h-4 h$ columns (and the single node) is $P_{1}=$

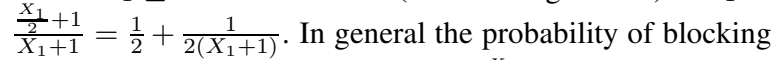
the even columns at step $i$ is $P_{i}=\frac{\frac{X_{i}}{2}+1}{X_{i}+1}=\frac{1}{2}+\frac{1}{2\left(X_{i}+1\right)}$, with $X_{i} \geq 2 h \geq 2$ (for $h=1$ ) for all steps except eventually the last one. Hence the probability of finding the global optimum by luckily removing the "right" nodes is at most $P=\left(\frac{1}{2}+\frac{1}{4 h+2}\right)^{\frac{1}{4} l h}=\left(\frac{1}{2}+\frac{1}{4 h+2}\right)^{\Omega(n)}$. One bit flip at a time is considered, however the probabilities of accepted flips of higher number of bits, leading towards the global optimum, are lower. If a 2-bit flip occurs, then the combinations of 2 nodes both of odd columns are very few compared to the total combinations of accepted node couples. For higher numbers of bit flips probabilities get even lower. However the number of steps required still depends on the problem size $n$.

Here we are optimistically considering that once the even columns are blocked then single bit flips will remove the remaining nodes. On the other hand, if an even column node is removed from the cover, then the probability a 2bit flip re-inserts it (event $E_{1}$ ) is $P_{E_{1}}=\frac{2 h}{n^{2}}\left(1-\frac{1}{n}\right)^{n-2}=$ $\frac{2(n-1)}{l(n-1)^{2}} \frac{1}{e}=\frac{2}{l(n-1) e}$. The probability that another node of the even column is removed (event $\left.E_{2}\right)$ is: $P_{E_{2}}=\frac{h-1}{n}(1-$ $\left.\frac{1}{n}\right)^{n-1} \geq \frac{h-1}{n e} \geq \frac{1}{2 l e}$. So the probability that event $E_{2}$ happens before $E_{1}$ is $P_{E_{2}-E_{1}} \geq \frac{P_{1}}{P_{1}+P_{2}}=\frac{1 /(2 l e)}{\frac{2}{l(n-1) e}+\frac{1}{2 l e}} \geq$ $1-\frac{4}{n}$. Hence $P_{E_{1}-E_{2}} \leq \frac{4}{n} \leq \frac{1}{2}+\frac{1}{4 h+2}$. If $E_{2}$ happens before $E_{1}$ then the probabilities of getting back on track towards the global optimum get even lower. In any case the total probability of reaching the global optimum before a 
local one is exponentially small.

Lemma 10: If the algorithm is initialised with an empty cover, then with overwhelmingly high probability, the $(1+1)$ EA does not find the global optimum before it finds a local search cover.

Proof: All the chosen nodes will be inserted in the cover except when they belong to columns that are adjacent to "full" columns both on the left and on the right. When this happens the column in the middle will be quickly emptied by using 1-bit flips. For the global optimum to be found, all the even columns have to be filled before all the adjacent odd ones. Similar reasoning to that of the previous lemma is used to prove that the probability is very low that flips of more than one bit invert a full column with one that is not full before the latter column is emptied. Given a block of four columns the probability that the even columns are completely filled before the odd ones is $P=\frac{1}{2}$. So the probability that this happens for all the blocks of four columns each is $P=$ $\left(\frac{1}{2}\right)^{\frac{1}{4} l h}=\left(\frac{1}{2}\right)^{\Omega(n)}$.

Lemma 11: If the algorithm is initialised with a uniform distribution, then with overwhelmingly high probability, the $(1+1)$-EA does not find the global optimum before it finds a local search cover.

Proof: The proof is similar to that of the previous lemma considering that, for large enough $h$, each column is initialised with at least a minimum number of nodes in the cover with overwhelming probability.

In the following theorem 5 is proved.

Proof: Once the EA has found a cover of at most size $\left\lfloor\frac{2}{3} l\right\rfloor h+1$, which is not the global optimum, the minimum number of bits that need to be flipped in order to leave the current position is $2 h$. Hence, the probability $P_{\text {move }}$ of each accepted move is at most: $P_{\text {move }}=\left(\frac{1}{n}\right)^{2 h}\left(1-\frac{1}{n}\right)^{n-2 h} \leq$ $\left(\frac{1}{n}\right)^{2 h}$. Such a probability implies an expected number of generations for each move of at least $n^{2 h}$ which leads to an expected time of $\Omega\left(n^{2 \sqrt{n-1}}\right)$ if $h=l$ and of $\Omega\left(n^{2 h}\right)$ if $l$ is a constant.

Theorems 4 and 5 show that given polynomial time the approximation ratio that can be guaranteed by the $(1+1)$-EA is roughly $4 / 3$, which is far away from optimality. However, in the following theorem it is proved that the $(1+1)$-EA still performs better than VERCOV on the instance class.

Theorem 6: With a probability of at most $O\left(2^{-n}\right)$, VERCOV produces a cover of size $\left\lceil\frac{2}{3} l\right\rceil h$ on the instance class graphs. With a probability of at least $1-O\left(2^{-n}\right)$, the cover size is larger than $\left\lceil\frac{2}{3} l\right\rceil h$.

The theorem implies that only when $l$ is a multiple of 3 is VERCOV able to produce a solution of equal size as that of the "worst local search cover" However this occurs only with exponentially low probability. If $l$ is not a multiple of 3 VERCOV always produces covers of larger size than those produced by the $(1+1)$-EA on the considered instance class (i.e. if $l \bmod 3=1$ the cover will be at least $\left\lfloor\frac{2}{3} l\right\rfloor h+2$, while if $l \bmod 3=2$ then the cover will be at least $\left.\left\lceil\frac{2}{3} l\right\rceil h\right)$.

Lemma 12: VERCOV is not able to construct an optimal cover or an almost perfect cover on graphs of the considered instance class.

Proof: Since the algorithm picks edges randomly and inserts both endpoints in the cover, all the nodes in the cover produced by VERCOV must be adjacent two by two. It follows that any cover having at least a node $i$ that is not adjacent to any other node in the cover can not be generated by VERCOV. Both the optimal cover and all the possible almost perfect covers belong to the described above category. Hence, the proof follows.

In the following Theorem 6 is proved.

Proof: VERCOV chooses randomly one edge at a time, and inserts both endpoints in the cover. Hence, the best solution VERCOV may produce is that having two columns in the cover out of every three adjacent columns. For VERCOV to produce a solution of $\left\lceil\frac{2}{3} l\right\rceil h$ nodes it has to choose only edges connecting nodes of adjacent twocolumn blocks which belong to the described cover and avoiding all edges connecting any node of the third column in a three adjacent column block. If a different edge is chosen, then the resulting cover will have a larger size. For each two-column block there are $h^{2}$ edges, and the number of blocks is $\left\lfloor\frac{1}{3} l\right\rfloor$. Except for the blocks on the left and right edges of the graph, for each group of three adjacent blocks, the algorithm has to pick $h$ edges from the middle block while avoiding to choose any edge from the other two blocks. For each of these three adjacent blocks the probability of picking the first edge out of the middle block is: $P_{0}=\frac{h^{2}}{3 h^{2}}=\frac{1}{3}$. Having chosen a "correct" first edge, for this group of blocks, the probability of choosing the next edge in the middle is: $P_{1}=\frac{(h-1)^{2}}{(h-1)^{2}+2\left(h^{2}-h\right)} \leq \frac{(h-1)^{2}}{3(h-1)^{2}} \leq \frac{1}{3}$. In general: $P_{i}=\frac{(h-i)^{2}}{(h-i)^{2}+2\left(h^{2}-i h\right)} \leq \frac{(h-i)^{2}}{3(h-i)^{2}} \leq \frac{1}{3}$. The far left and far right groups of blocks form an exception since they respectively do not have a set of $h^{2}$ edges on the left or on the right. For the far left the probability of choosing a correct first edge is $P_{0}=\frac{h^{2}}{2 h^{2}}$, and in general: $P_{i}=\frac{(h-i)^{2}}{(h-i)^{2}+\left(h^{2}-i h\right)} \leq \frac{(h-i)^{2}}{2(h-i)^{2}} \leq \frac{1}{2}$. While for the far right blocks the probabilities for edge $i$ of the "right" block of being chosen is: $P_{i}=\frac{(h-i)^{2}}{(h-i)^{2}+\left(h^{2}-i h\right)+h} \leq \frac{(h-i)^{2}}{2(h-i)^{2}+h} \leq \frac{1}{2}$. Each group of three adjacent blocks is independent from the other. So the probability of choosing all the $h$ edges from each block is less than $\left(\frac{1}{2}\right)^{h}$ and since the blocks are $\left\lfloor\frac{1}{3} l\right\rfloor$, the probability of reaching the cover, without choosing any of the "outside" edges, is: $P_{l} \leq\left(\frac{1}{2}\right)^{h\left\lfloor\frac{1}{3} l\right\rfloor}=O\left(2^{-n}\right)$. Hence the expected number of times VERCOV should be run before it produces such a cover is at least $\Omega\left(2^{n}\right)$. With probability $1-O\left(2^{n}\right)$ VERCOV will produce a cover of larger size.

\section{THE BIPARTITE INSTANCE CLASS}

In [15], the (1+1)-EA and a global Simple Evolutionary Multi-objective Optimizer (SEMO) have been recently analysed on a bipartite instance class. The instance is depicted in figure 5. In the paper it is proved that for $n^{\delta-1} \leq \epsilon<1 / 2$ and $\delta>0$ the $(1+1)$-EA has an expected optimisation time which is exponential to produce an approximation that is better than $\frac{1-\epsilon}{\epsilon}$. In the following the $(1+1)$-EA is further 


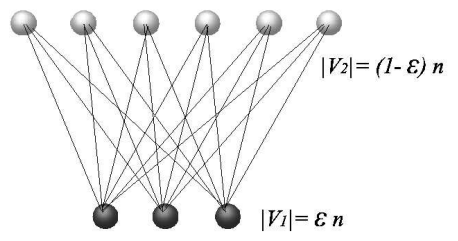

Fig. 5. The optimal cover of the bipartite graph with $n=9$ and $\epsilon=\frac{1}{3}$.

analysed and it is pointed out that, by using multiple runs, the $(1+1)$-EA finds the optimal solution.

Theorem 7: Let $\epsilon=\frac{i}{n}$ with $1<i<\frac{n}{2}$. The expected optimisation time of the (1+1)-EA to find the optimal solution of the bipartite graph is $O\left(n^{2 i}\right)$. With probability at least $1 / 2 e$ the $(1+1)$-EA finds the global optimum in time $O(n \log n)$

Proof: For the given values of $\epsilon,\left|V_{1}\right|=i$ and $\left|V_{2}\right|=n-i$. It has already been proved in [15] that in time $O(n \log n)$ the $(1+1)$-EA will find a cover. Furthermore, in a similar manner as for the other instances analysed in the present paper, it is proved that the $(1+1)$-EA will find a local search cover in expected time $O(n \log n)$. For the bipartite graph there are only two local search covers: $C_{1}$ containing all the $V_{1}$ nodes and $C_{2}$ containing all the $V_{2}$ nodes. If the algorithm finds $C_{1}$ before finding $C_{2}$, then obviously it has found the global optimum. With probability at least $1 / 2$ in $O(n \log n)$ time and whatever the initialisation, all the $V_{1}$ nodes are in the current cover while all the $V_{2}$ nodes are not. The conditional probability of finding the global optimum, given the above event, is higher than $\frac{1}{e}$ for the same reasons as discussed in the proof of theorem 2. If, instead, $C_{2}$ is found first, then the time to reach $C_{1}$ from $C_{2}$ has to be analysed. Once $C_{2}$ has been obtained the only accepted moves will be those flipping all the $V_{1}$ nodes together with the same amount of nodes belonging to $V_{2}$. The probability for the event to happen is $\Omega\left(n^{-2 i}\right)$ giving an expected time of $O\left(n^{2 i}\right)$. Once the $V_{1}$ bits have been inverted, with probability greater than $\frac{1}{e}$ the other $V_{2}$ nodes will be removed from the cover in $O(n \log n)$ time.

\section{CONCLUSIONS}

Theoretical proofs of how and why the $(1+1)$-EA performs better than VERCOV on the previously examined instance classes have been presented. Given polynomial time the $(1+1)$-EA outperforms VERCOV in solution quality even when it is not able to find the optimal cover on the given graphs. However, an analysis on a bipartite graph class proves that only if multiple runs of the $(1+1)$-EA are used can polynomial time poor approximations be avoided. Hence the next question to be answered is what approximate solution can be guaranteed by the $(1+1)$-EA whatever the instance class if multiple runs are used. A lower bound of roughly $\frac{4}{3}$ on the approximation quality that can be guaranteed by the (1+1)-EA using restarts is given in the analysis of the exponential time instance class. However, such a bound may not be tight. Another direction for future research is to consider populations rather than single individuals, with and without crossover.

\section{ACKNOWLEDGMENT}

This work was supported in part by an EPSRC grant (EP/C520696/1). The authors are grateful to Dr. Per Kristian Lehre and to Dr. Carsten Witt for insightful discussions.

\section{REFERENCES}

[1] R. Sarker, M. Mohammadian, and X. Yao, Eds., Evolutionary Optimization. Norwell, MA, USA: Kluwer Academic Publishers, 2002.

[2] G. Rudolph, "Finite Markov chain results in evolutionary computation: A tour d'horizon," Fundamenta Informaticae, vol. 35, no. 1-4, pp. 6789, 1998. [Online]. Available: citeseer.ist.psu.edu/rudolph98finite.html

[3] S. Droste, T. Jansen, and I. Wegener, "On the optimization of unimodal functions with the $(1+1)$ evolutionary algorithm," in PPSN V: Proceedings of the 5th International Conference on Parallel Problem Solving from Nature. London, UK: Springer-Verlag, 1998, pp. 13-22.

[4] T. Jansen and I. Wegener, "Evolutionary algorithms: How to cope with plateaus of constant fitness and when to reject strings of the same fitness," IEEE Transactions on Evolutionary Computation, vol. 5, no. 6, pp. 589-599, 2001

[5] I. Wegener, "Methods for the analysis of evolutionary algorithms on pseudo-boolean functions," in Evolutionary Optimization, R. Sarker, M. Mohammadian, and X. Yao, Eds. Dordrecht, The Netherlands: Kluwer Academic Publishers, 2001.

[6] J. He and X. Yao, "Towards an analytic framework for analysing the computation time of evolutionary algorithms," Artif. Intell., vol. 145, no. 1-2, pp. 59-97, 2003.

[7] O. Giel and I. Wegener, "Evolutionary algorithms and the maximum matching problem," in STACS '03: Proceedings of the 20th Annual Symposium on Theoretical Aspects of Computer Science. London, UK: Springer-Verlag, 2003, pp. 415-426.

[8] F. Neumann and I. Wegener, "Randomized local search, evolutionary algorithms, and the minimum spanning tree problem," in Proceedings of GECCO 2004, LNCS 3102. Springer, 2004, pp. 713-724.

[9] C. Witt, "Worst-case and average-case approximations by simple randomized search heuristics," in Proc. of the 22nd Annual Symposium on Theoretical Aspects of Computer Science (STACS '05), LNCS 3404. Springer, 2005, pp. 44-56.

[10] J. He and X. Yao, "Drift analysis and average time complexity of evolutionary algorithms," Artificial Intelligence, vol. 127, no. 1, pp. 57-85, 2001. [Online]. Available: citeseer.ist.psu.edu/he00drift.html

[11] — "A study of drift analysis for estimating computation time of evolutionary algorithms," Natural Computing: an international journal, vol. 3, no. 1, pp. 21-35, 2004.

[12] S. Khuri and T. Bäck, "An evolutionary heuristic for the minimum vertex cover problem," in Genetic Algorithms within the Framework of Evolutionary Computation - Proc. of the KI-94 Workshop, J. Hopf, Ed., Saarbrücken, Germany, 1994, pp. 86-90.

[13] K. S. Christos H. Papadimitriou, Combinatorial Optimization: Algorithms and Complexity. Dover Publications Inc., 1982.

[14] J. He, X. Yao, and J. Li, "A comparative study of three evolutionary algorithms incorporating different amounts of domain knowledge for node covering problem." IEEE Transactions on Systems, Man, and Cybernetics, Part C, vol. 35, no. 2, pp. 266-271, 2005.

[15] T. Friedrich, J. He, N. Hebbinghaus, F. Neumann, and C. Witt, "Approximating covering problems by randomized search heuristics using multi-objective models," in Proceedings of the Genetic and Evolutionary Computation Comference (GECCO2007), London, July 2007.

[16] S. Droste, T. Jansen, and I. Wegener, "A rigorous complexity analysis of the $(1+1)$ evolutionary algorithm for separable functions with boolean inputs." Evolutionary Computation, vol. 6, no. 2, pp. 185196, 1998.

[17] P.S.Oliveto, J. He, and X. Yao, "Evolutionary algorithms and the vertex cover problem," School of computer science, University of Birmingham, Tech. Rep. No. CSR-07-5, June 2007.

[18] W. Feller, An introduction to probability theory and its applications. - Vol. 1. Wiley, 1968. 